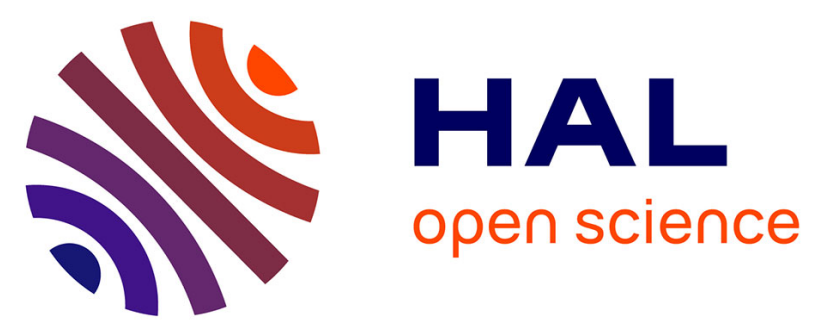

\title{
Organogel Formation Rationalized by Hansen Solubility Parameters: Shift of the Gelation Sphere with the Gelator Structure
}

\author{
Danilo Rosa Nunes, Manuel Reche-Tamayo, Emilie Ressouche, Matthieu \\ Raynal, Benjamin Isare, Pascale Foury-Leylekian, Pierre-Antoine Albouy, \\ Patrick Brocorens, Roberto Lazzaroni, Laurent Bouteiller
}

\section{To cite this version:}

Danilo Rosa Nunes, Manuel Reche-Tamayo, Emilie Ressouche, Matthieu Raynal, Benjamin Isare, et al.. Organogel Formation Rationalized by Hansen Solubility Parameters: Shift of the Gelation Sphere with the Gelator Structure. Langmuir, 2019, 35 (24), pp.7970-7977. 10.1021/acs.langmuir.9b00966 . hal-02285236

\section{HAL Id: hal-02285236 \\ https://hal.sorbonne-universite.fr/hal-02285236}

Submitted on 12 Sep 2019

HAL is a multi-disciplinary open access archive for the deposit and dissemination of scientific research documents, whether they are published or not. The documents may come from teaching and research institutions in France or abroad, or from public or private research centers.
L'archive ouverte pluridisciplinaire HAL, est destinée au dépôt et à la diffusion de documents scientifiques de niveau recherche, publiés ou non, émanant des établissements d'enseignement et de recherche français ou étrangers, des laboratoires publics ou privés. 


\section{Organogel formation rationalized by Hansen solubility parameters: shift of the gelation sphere with the gelator structure}

Danilo Rosa Nunes, ab Manuel Reche, ac Emilie Ressouche, d Matthieu Raynal, a Benjamin Isare, ,

Pascale Foury-Leylekian, ${ }^{b}$ Pierre-Antoine Albouy*, " Patrick Brocorens, ${ }^{,}$Roberto Lazzaroni*, and Laurent Bouteiller*a

a Sorbonne Université, CNRS, Institut Parisien de Chimie Moléculaire (IPCM), Equipe Chimie des Polymères, F-75005 Paris, France.

E-mail: laurent.bouteiller@sorbonne-universite.fr

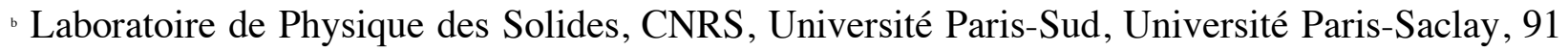
400, Orsay, France

E-mail: pierre-antoine.albouy@u-psud.fr

- Service de Chimie des Matériaux Nouveaux, Institut de Recherche en Science et Ingénierie des Matériaux, Université de Mons, Place du Parc, 20, B-7000 Mons, Belgium

E-mail: roberto.lazzaroni@umons.ac.be

A Aalto University, Department of Applied Physics, P.O. Box 15100, FIN-00076 Aalto, Espoo, Finland.

KEYWORDS.

organogel, powder XRD, crystal habit, Hansen solubility parameters 


\section{ABSTRACT}

In order to rationalize how the gelation ability of a low molecular weight gelator is influenced by its molecular structure, we performed extensive solubility tests of a group of thiazole based gelators and made use of Hansen solubility parameters formalism. We observe that the increase of a linear alkyl chain in these gelators promotes an increase of the radius of the gelation sphere as well as a gradual shift of its center to lower values of the polar $\left(\delta_{\mathrm{P}}\right)$ and hydrogen bonding $\left(\delta_{\mathrm{H}}\right)$ components. The molecular packing within the fibers and the crystal habit were determined by a combination of X-ray diffraction and molecular modeling. We attribute the gradual and linear shift of the gelation sphere to the fact that all the studied gelators share the same molecular packing, so that an increasing length of the alkyl chain reduces the proportion of polar groups at the surface, resulting in a gradual increase in the contact between apolar parts of the fiber and the solvent.

\section{Introduction}

Organogels are solid-like materials resulting from the self-assembly of low molecular weight gelators (LMWG) into anisotropic structures within a liquid. ${ }^{1-3}$ These anisotropic structures (usually fibrillar) entrap the liquid altering the mechanical properties of the system.

Several examples of LMWG have been reported in the literature showing a high structural variety between them with different levels of complexity. Such molecules are usually discovered by serendipity, and their gelation properties studied by time-consuming gelation tests. In an attempt to speed up this process we $\mathrm{e}^{4-6}$ and others ${ }^{7-26}$ have applied the Hansen solubility parameter (HSP) framework to try and rationalize the gelation proprieties of several LMWG families. To apply this methodology, we need to explore the Hansen space defined by the three HSP cohesive 
energies of the tested liquids: dispersive interactions $\left(\delta_{\mathrm{p}}\right)$, polar interactions $\left(\delta_{\mathrm{p}}\right)$ and hydrogen bonds $\left(\delta_{н}\right)$. Following the exploration of the Hansen space, it is possible to determine a gelation domain for individual LMWGs. This can then be used to predict whether an untested liquid could be gelated by a particular LMWG. ${ }^{4}$

The next challenge in the field is to evaluate how small changes in the structure of a LMWG will affect the gelation domain. ${ }^{27-33}$ In particular, we previously probed the influence of the length of an alkyl chain within a family of related gelators, but showed that it is difficult to rationalize the results without knowledge of the molecular packing. ${ }^{33}$

To better correlate the molecular structure with the gelation domain, we selected a family of amide-based thiazole derivatives bearing a long aliphatic chain, for which the elucidation of the molecular packing seemed to be within reach (Figure 1). Within these gelators the amide group is expected to provide supramolecular H-bond interactions while the long alkyl chain contributes to the dispersion interactions. This group of LMWGs was first reported by Ballabh et al. together with the single-crystal structure of two members of the family ${ }^{34,35}$. In the present work, the gelation domains of several members of this family, with the alkyl chain length ranging from 6 to $16 \mathrm{CH}_{2}$ groups, are determined, together with structural characterization (X-ray diffraction measurements and scanning electronic microscopy). To extract detailed structural information from the X-ray powder patterns, a molecular modeling protocol is applied, in which the most probable packing structure is identified by comparing the simulated patterns of dozens of candidates to the experimental data. The crystal habit is then evaluated using the Bravais-Friedel-Donnay-Harker method $^{36,37}$, and the Growth Morphology Method ${ }^{38,39}$ (GMM) based on attachment energy of the crystal faces, in order to determine along which direction crystalline fibers are expected to grow 
preferentially. With this study, we thus aim at correlating the gelation domain with the molecular packing and the fiber morphology of the gels.

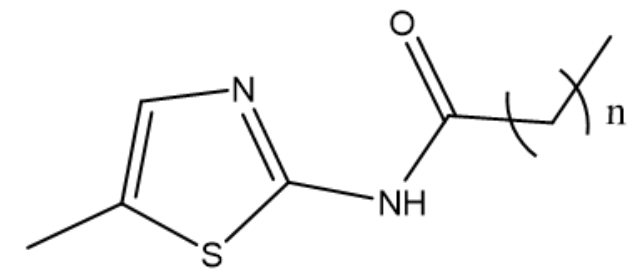

$$
\begin{array}{ll}
\text { Th8 } & \mathrm{n}=6 \\
\text { Th12 } & \mathrm{n}=10 \\
\text { Th14 } & \mathrm{n}=12 \\
\text { Th16 } & \mathrm{n}=14 \\
\text { Th18 } & \mathrm{n}=16
\end{array}
$$

Figure 1. Chemical structure of the thiazole-based LMWGs.

\section{Experimental section}

\section{Gel preparation}

The samples were prepared by adding $20 \mathrm{mg}$ of LMWG and $1 \mathrm{~mL}$ of liquid in a screw-cap vial. The suspensions were heated up to $100^{\circ} \mathrm{C}$ until dissolution and left to cool to room temperature on the bench. After 24 hours the vials were turned upside-down, and the aspect of the samples was noted as gel $(\mathrm{G})$, solution $(\mathrm{S})$ or precipitate $(\mathrm{P})$. A material is considered a gel if no deformation is observed after turning the vial upside-down.

\section{Hansen sphere determination}

All solubility and gelation spheres were calculated according to our recently proposed general procedure, ${ }^{6}$ using the generic algorithm provided in the HSPiP software ${ }^{40,41}$ In short, the solubility sphere was calculated in a way that as many S points as possible lie inside the sphere, but as many $\mathrm{G}$ and $\mathrm{P}$ points as possible lie outside. The gelation sphere was determined so that as many $\mathrm{G}$ points as possible lie inside the sphere, but as many P points as possible lie outside. S points are not taken in consideration for the gelation sphere.

\section{X-ray diffraction measurements}


Xerogels were obtained by extracting gel fibers by filtration of a gel and drying overnight. The measurements were performed with a Rigaku SmartLab X-Ray Diffractometer using a rotating $\mathrm{Cu}$ anode X-ray source $(40 \mathrm{kV}, 200 \mathrm{~mA})$.

\section{Modeling of the molecular packing}

As the first step, the X-ray patterns were indexed using Dicvol ${ }^{42}$ and the symmetry groups were obtained using the Check group program incorporated in the FullProf suite. ${ }^{43}$ In parallel, the atomic positions were obtained by molecular modeling simulations, through Simulated Annealing using the Polymorph module, implemented in the Materials Studio software. ${ }^{44}$ The generated molecular packings were then optimized by Molecular Mechanics (MM). All the MM calculations were performed with the Dreiding force field (in which the hydrogen bond parameters have been adapted to properly reproduce van der Waals interactions in the solid state) and assigning PCFF atomic charges. The best models were then refined using the Reflex module from the Materials Studio software by fitting the experimental XRD pattern using Rietveld refinement coupled with the force field to avoid unrealistic changes in the molecular geometry. This joint modelingexperimental approach (see SI for more technical details) has been fully implemented for the Th12 compound, and the agreement between the simulations and the single crystal X-ray data has been found to be excellent (see below). That structure was then used as a starting point for determining the crystal structure of the other members of the series, by increasing the chain length, adapting the cell parameter along the chain direction, and refining the structure using Reflex.

\section{Crystal habit calculation}

The calculations were performed in the Materials Studio software on crystals that were fully relaxed (including the cell parameters) by MM using the modified Dreiding force field. Due to the force field approximations, this full relaxation slightly changes the cell parameters of the proposed 
cell but ensures that energy discrepancies are avoided (the repulsive wall of van der Waals interactions is a few tenths of $\AA$ far from the minimum). The long-range interactions were treated by Ewald for electrostatics (accuracy of $10^{-5} \mathrm{kcal} / \mathrm{mol}$ ) and an atom-based cutoff for van der Waals (cutoff distance of $14 \AA$, spline width of $3 \AA$ ).

\section{Scanning electron microscopy (SEM)}

The samples for SEM observation (Zeiss Sigma VP microscope) were prepared by freeze-drying the gel samples in liquid nitrogen and vacuum-pumping the solvent.

\section{Results and discussion}

\section{Determination of the gelation domain}

First, the gelation domain in Hansen space of LMWG Th12 was determined using our previously established methodology. ${ }^{6}$ The gelator concentration was varied between 3 and $0.75 \mathrm{wt} \%$ (Figure S1). We observed that: (i) below $1 \mathrm{wt} \%$ Th12 formed very few gels in the tested liquids and (ii) at $3 \mathrm{wt} \%$ almost none of the tested liquids fully solubilized the LMWG. Therefore, we selected an intermediate concentration ( $2 \mathrm{wt} \%)$ to be able to detect any effect of the length of the alkyl chain on the gelation domain. The gelation domain of LMWGs Th8 to Th18 was then determined using the same methodology at the concentration of $2 \mathrm{wt} \%$ (Figure 2). 
Th 8

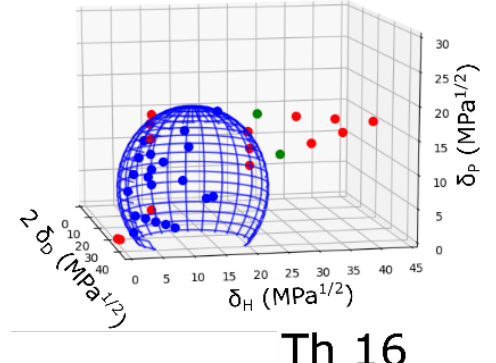

Th 12

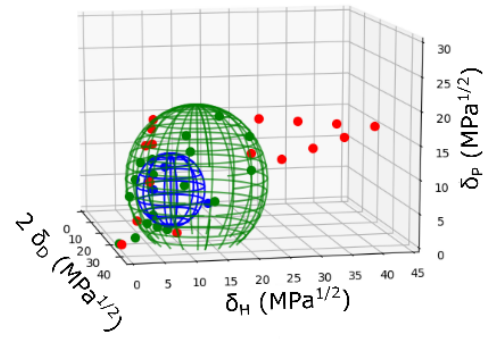

Th 14

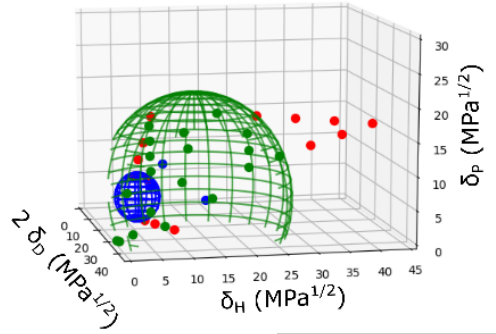

Th 18
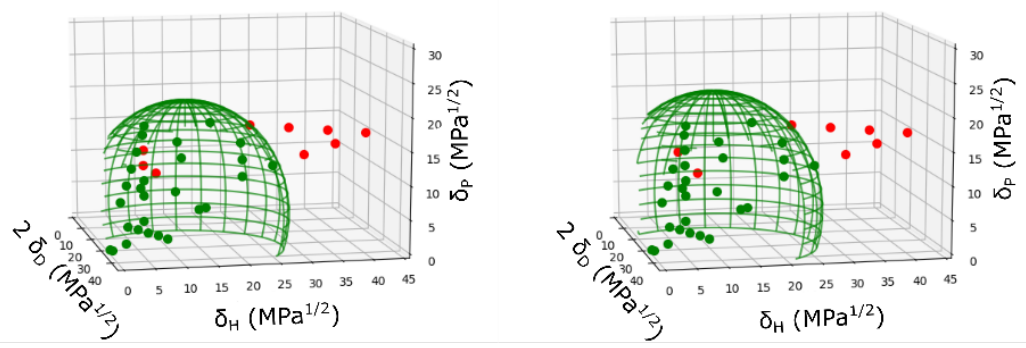

Figure 2. Gelation data plotted in Hansen space for the thiazole-based LMWGs at 2 wt $\%$. The tested liquids are represented by full circles and the calculated domains are represented by meshed spheres. Green: gel; red: precipitate; blue: soluble. ${ }^{45}$

The evolution of the size of the solubility and gelation spheres is summarized in Figure 3a. The solubility sphere dramatically shrinks with the alkyl chain length, in such a way that for Th16 and Th18, solubility is no longer observed. In clear contrast, the increase in length of the alkyl chain leads to an expansion of the gelation sphere from Th12 to Th18. ${ }^{45}$

As expected, the center of the solubility sphere shifts gradually to a less polar region (smaller $\delta_{\text {н }}$ and $\delta_{\mathrm{p}}$ ) and to a less polarisable region of the Hansen space (smaller $\delta_{\mathrm{p}}$ ) as the alkyl chain length increases (Figure 3b). More interestingly, a very similar trend also occurs for the gelation sphere, which is in contrast to our previous study. ${ }^{33}$ It can be seen from the trend lines (see SI) that adding one $\mathrm{CH}_{2}$ decreases the parameters for the center of the solubility sphere by $2 \delta_{\mathrm{D}}=0.43 ; \delta_{\mathrm{P}}=0.25$; $\delta_{\mathrm{H}}=0.40 \mathrm{MPa}^{1 / 2}$, while it decreases the parameters for the center of the gelation sphere by $2 \delta_{\mathrm{D}}=$ $0.51 ; \delta_{\mathrm{P}}=0.23 ; \delta_{\mathrm{H}}=0.41 \mathrm{MPa}^{1 / 2}$. Therefore, a longer alkyl chain increases the contribution of the 
apolar group with respect to the contribution of the polar one, both on the solubility sphere and on the gelation sphere. Finally, one can notice that the shift of the center of the gelation sphere to a less polar region is not accompanied by a loss of gelation in the polar region because the shift in the center occurs together with the increase of the radius of the gelation sphere.
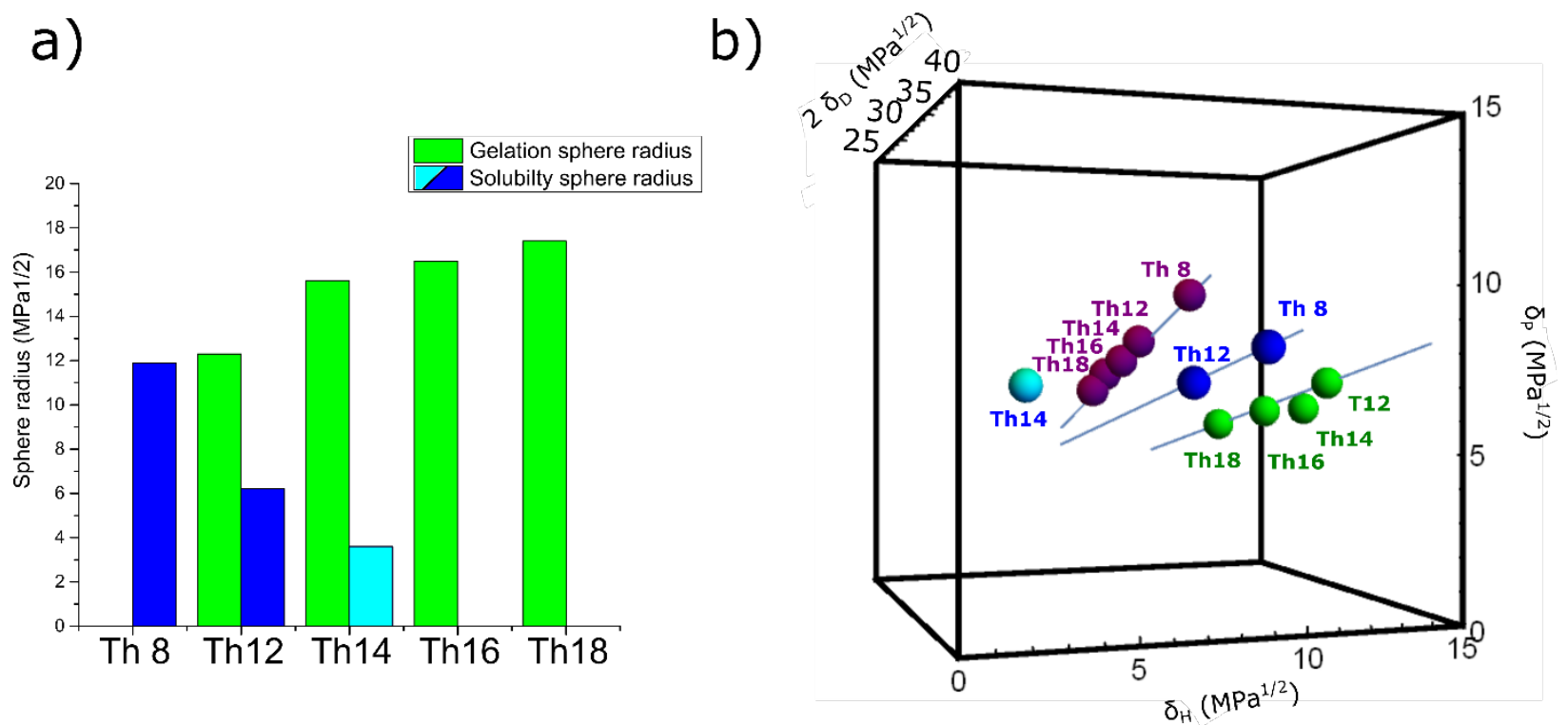

Figure 3. a) Radius of the solubility (blue) or the gelation (green) spheres for the thiazole-based LMWGs. b) Center of the solubility spheres (blue: experimental; purple: calculated according to HSPiP software. ${ }^{40,41}$ ) and center of the gelation spheres (green) represented in Hansen space. Data from Figure 2; for Th14, a light blue color has been selected to highlight the limited precision of that particular data (only $4 \mathrm{~S}$ points); the lines are drawn as explained in SI.

In order to try and rationalize these observations, we investigated the morphology and the structure of the fibers. 


\section{SEM observation of fibers}

The morphology of Th12 to Th18 gel fibers was observed by scanning electronic microscopy (SEM). Th12 xerogels obtained from various liquids (toluene, cyclohexane and 1-butanol) (Figure 4.a to c) all show rectilinear shaped fibers with very few differences in size, indicating that the different liquids do not affect the morphology of the fibers. Moreover, the fibers of the various gelators Th12 to Th18 (Figure 4.a and d to f) obtained from toluene also share a very similar morphology, even if the Th18 sample appears more blurred than the others. Therefore, no major effect is detected at this length scale.
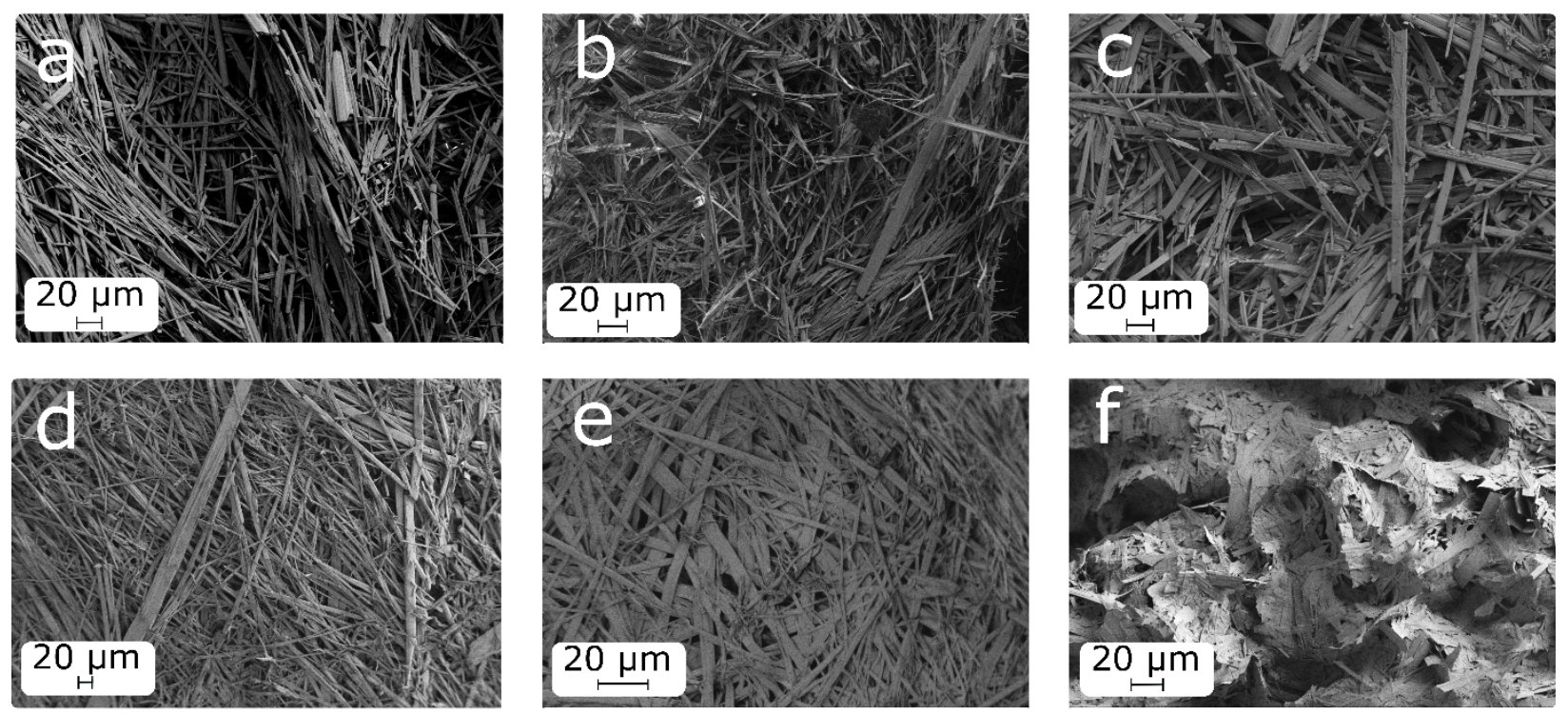

Figure 4. SEM images of the thiazole-based xerogels: $a$ - Th12 from toluene, $b$ - Th12 from cyclohexane, c - Th12 from 1-butanol, d - Th14 from toluene, e - Th16 from toluene, f - Th18 from toluene. 


\section{X-ray measurements in transmission}

X-ray measurements were performed on xerogels obtained from Th12 to Th18 from various liquids to determine whether the nature of the liquid influences the packing in the fibers. Xerogels were grinded to obtain a fine powder and then measured in transmission mode with the X-ray beam set-up in the parallel beam configuration. The data from the Th12 and Th14 xerogels were compared to the powder pattern simulated from the previously published single-crystal structure. ${ }^{34}$

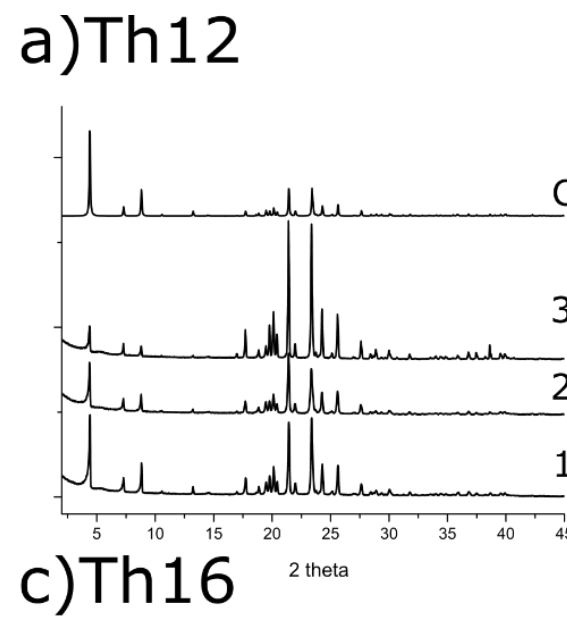

b)Th14
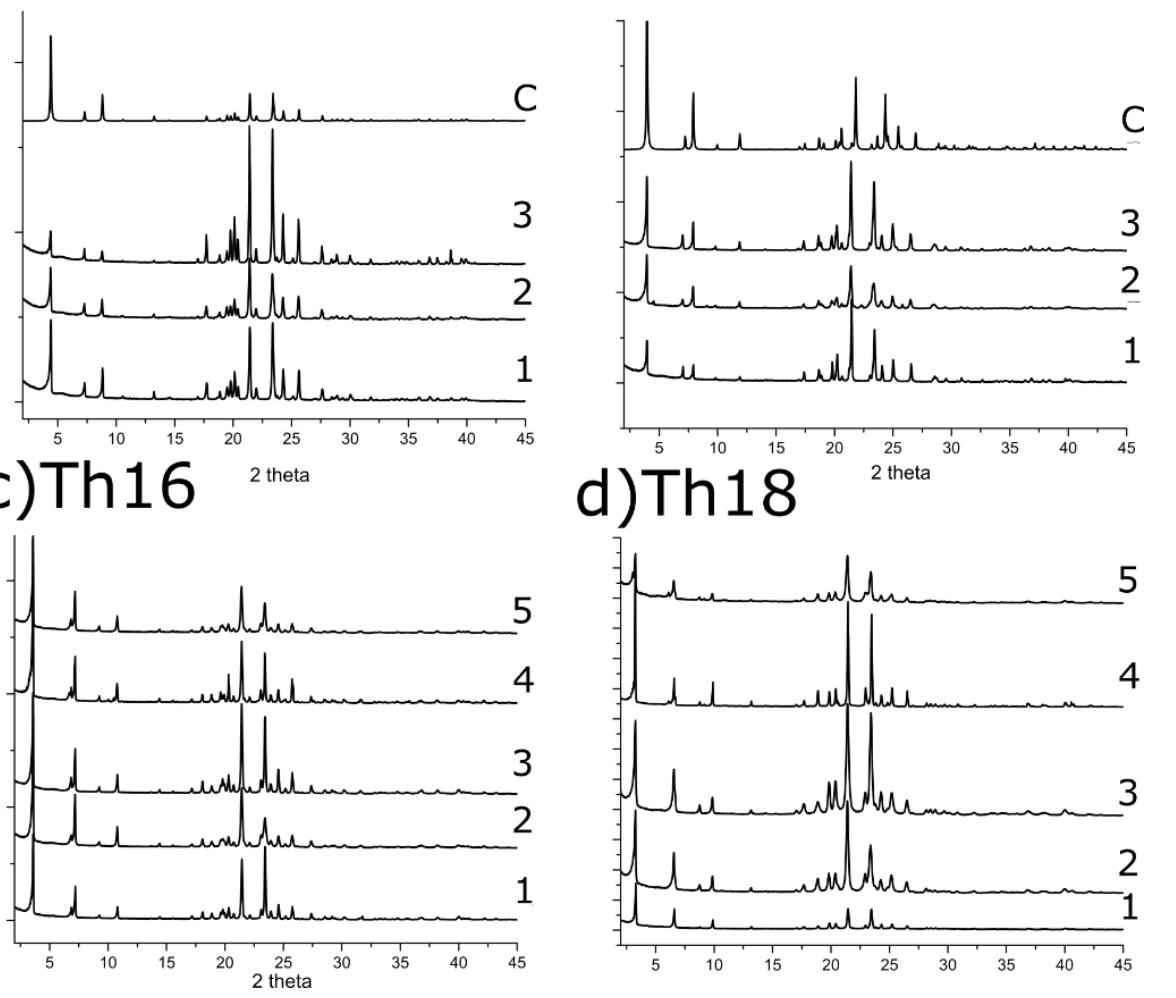

Figure 5. X-ray patterns of xerogels of the thiazole-based gelators obtained from (1) toluene, (2) cyclohexane, (3) 1-butanol, (4) acetonitrile, (5) 1,4-dioxane. Pattern simulated from the singlecrystal structure (C).

Similar X-ray powder patterns were obtained from the Th12 xerogels regardless of the liquid in which they were formed (Figure 5a 1,2,3). Small differences in diffraction peak intensity can be 
attributed to small effects of preferential orientation within the samples. This observation also applies to Th14, Th16, and Th18 (Figure 5b, c, d). This indicates that in this family of LMWGs, the molecular packing within the fibers is independent of the liquid in which the gel is formed. In fact, different patterns for a given gelator were only observed for Th12 when precipitates were obtained instead of gels (Figure S2). Thus, within each gelation sphere the molecular packing can be considered to be similar. The xerogels obtained from Th12 also present a high resemblance with the powder pattern simulated from the published single-crystal structure of Th12 (Figure 5a C) $)^{34}$. This indicates that the gel fibers of Th12 share the same packing as the corresponding singlecrystal.

Th14 shows the same X-ray pattern for all the measured xerogels (Figure 5b 1,2,3) but small differences appear with respect to the powder pattern simulated from the single-crystal data ${ }^{34}$ (Figure $5 \mathrm{~b} \mathrm{C}$ ): the measured xerogels show a good match to the single-crystal pattern in the small angle region ( 0 to 15 degrees) and a constant small shift of +0.5 degrees in the wide-angle region (above 15 degrees). This small shift is due to a slightly denser packing (3.9\%) in the case of the single crystal (see Table S9), but the conformations are identical and the molecular packings are very similar (see below and Figure S4).

\section{Modeling of the molecular packing}

To better understand how the length of the alkyl chain affects the crystal structure within the gel fibers, the molecular packing was determined based on the powder patterns measured on the xerogels and molecular modeling simulations. The validation of the methodology was performed by comparing the structure obtained for Th12 with the published single-crystal structure of the same molecule. Overlaying the two structures, it was found that they are similar, with cell parameters differing by less than $0.05 \AA$ and $0.1^{\circ}$, and atoms overlapping almost perfectly (Figure 
S3). The very good agreement between the modelled and published single-crystal structure validates the applicability of the established procedure to determine the molecular packing within the gel fibers. The structure proposed for the Th14 xerogels also presents a high resemblance with the previously published single-crystal structure (Figure S4). The indexation of the powder patterns for all these thiazole-based gelators points to monoclinic unit cells, with the cell parameter " $a$ " increasing with the length of the alkyl chain and the other cell parameters remaining constant (see Table 1). Figure 6 illustrates the high structural similarity between gelators Th12 to Th18, suggesting that differences in the length of the alkyl chain do not change the packing of these gelators within the gel fibers. The structures are characterized by the presence of $\mathrm{H}$-bonded dimers forming a cyclic H-bond topology (see Figures S7 and S8), where the amide group is the donor of hydrogen bond and the nitrogen of thiazole is the acceptor ( $\mathrm{NH} \ldots \mathrm{N}$ bonds), as reported previously ${ }^{34}$. The thiazole moieties of the dimers form planar objects that organize in a $\gamma$ packing, i.e., a combination of a parallel-displaced arrangement along the [001] direction and a herringbone arrangement along the [010] direction (see Figure S7). The sulfur atoms of neighboring stacks are in van der Waals contact, with a distance of $3.57 \AA$. All alkyl chains organize in an extended all-trans conformation, interdigitating along the (100) plane (Figure 6). The detailed structures and fitted XRD patterns are in Supporting Information. 


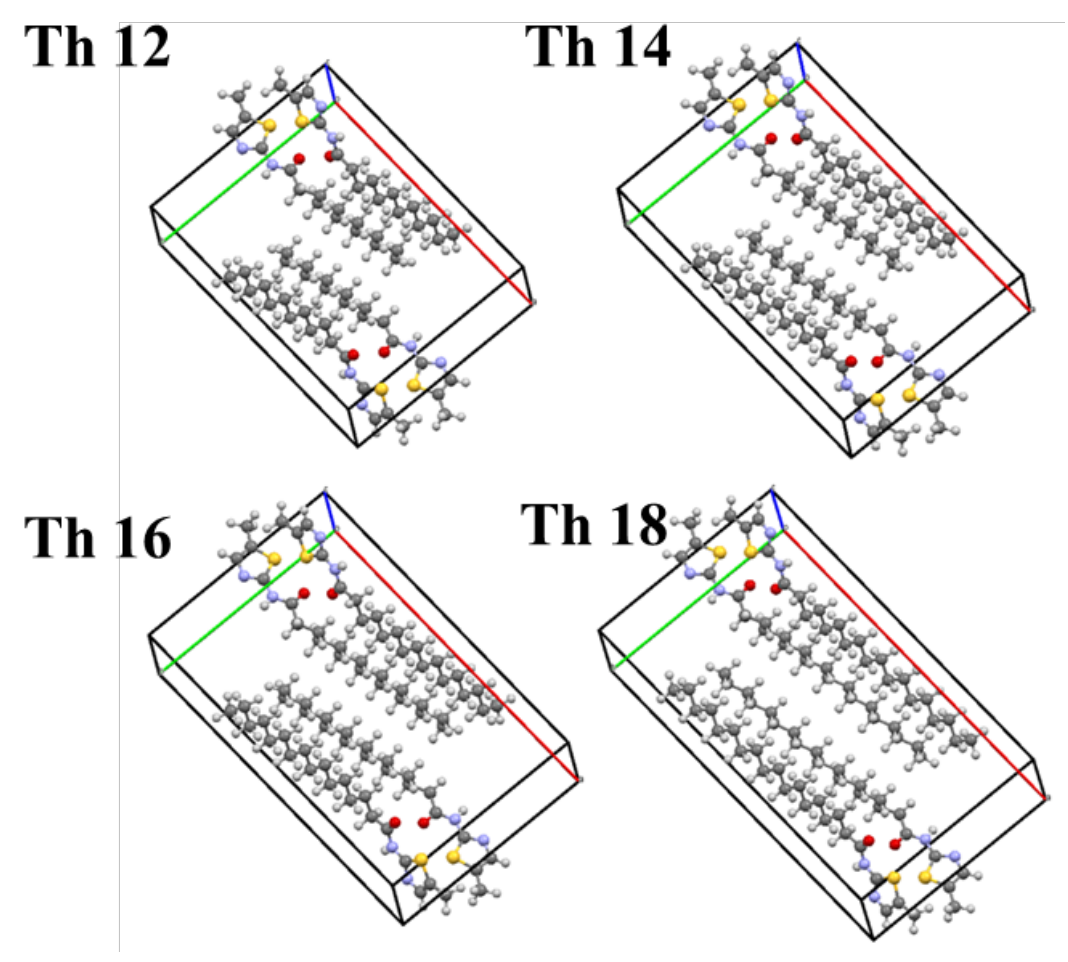

Figure 6. Molecular packing representation, with the cell parameters $a$ in red, $b$ in green, $c$ in blue.

Table 1. Proposed unit cell parameters of the thiazole-based gelators.

\begin{tabular}{ccccc}
\hline compound & Th12 & Th14 & Th16 & Th18 \\
\hline crystal system & monoclinic & monoclinic & monoclinic & monoclinic \\
\hline space group & $\mathrm{P} 2{ }_{1} / c$ & $\mathrm{P} 2{ }_{1} / c$ & $\mathrm{P} 2{ }_{1} / c$ & $\mathrm{P} 2{ }_{1} / c$ \\
\hline$a(\AA)$ & 20.00 & 22.30 & 24.60 & 26.99 \\
\hline$b(\AA)$ & 15.17 & 15.18 & 15.19 & 15.19 \\
\hline$c(\AA)$ & 5.54 & 5.55 & 5.55 & 5.56 \\
\hline$\alpha(\mathrm{deg})$ & 90 & 90 & 90 & 90 \\
\hline$\beta(\mathrm{deg})$ & 92.37 & 90.86 & 93.54 & 95.74 \\
\hline$\gamma(\mathrm{deg})$ & 90 & 90 & 90 & 90 \\
\hline volume $\left(\AA^{3}\right)$ & 1697.40 & 1889.64 & 2081.88 & 2274.12 \\
\hline $\mathrm{Z}$ & 4 & 4 & 4 & 4 \\
\hline density $\left(\mathrm{gcm}^{-3}\right)$ & 1.173 & 1.147 & 1.132 & 1.115 \\
\hline
\end{tabular}




\section{X-ray measurements on oriented fibers}

Usually, a strong directional interaction is responsible for the anisotropic growth of the organogel fibers. Here, this feature is not obvious since the amide groups are involved in dimer formation instead of chains of hydrogen bonds. To unambiguously identify which axis of the unit cell lies along the fiber growth direction, we performed X-ray diffraction on oriented fibers. By keeping the gel fibers intact (i.e., no grinding was performed) and compacting them as a film on a flat surface, we expect that most fibers lie parallel to the substrate. In these conditions, only reflections associated to lattice planes parallel to the fiber axis can be visualized in symmetric diffraction geometry (see Figure 7, which shows the difference in the experimental setup between the measurements performed on randomized crystals and oriented fibers).

Transmission
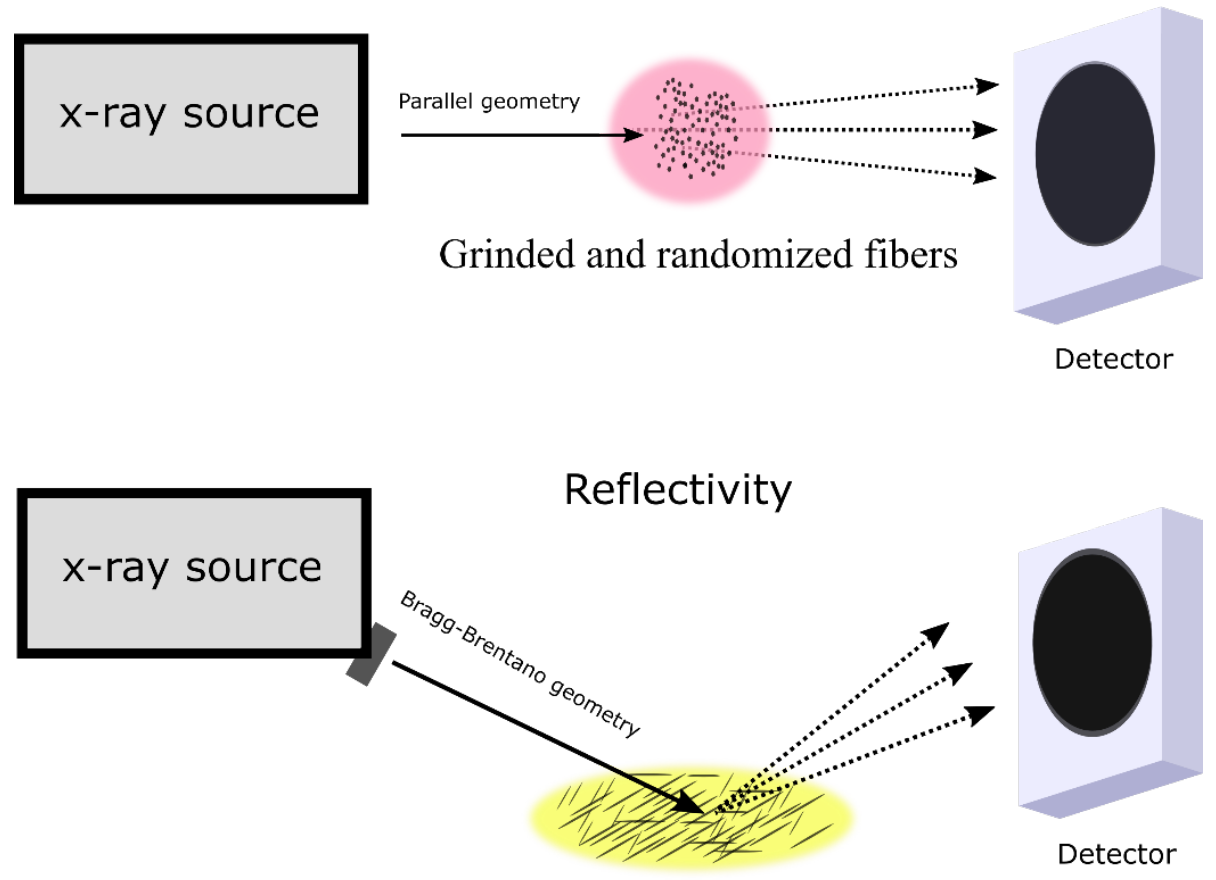

Oriented fibers

Figure 7. Comparison of the X-ray methodology to measure randomized or oriented gel fibers. 
Figure 8 illustrates the difference between the patterns obtained from randomized and oriented fibers for Th12 in toluene. The diffraction peaks that are present in both patterns can be easily identified as due to diffraction by (hk0) lattice planes. Reflections of the type 00L corresponding to the diffraction of the $(00 \mathrm{~L})$ planes are not present in the oriented fibers pattern. Thus the $(00 \mathrm{~L})$ planes are never in reflection condition which means that the $[00 \mathrm{~L}]^{*}$ direction, which deviates from the $[00 \mathrm{~L}]$ axis by only few degrees, is never orthogonal to the sample plane. This is an indication of a preferred orientation of the fibers in a $2 \mathrm{D}$ powder, where the $[00 \mathrm{~L}]$ axis is in the sample plane, and should thus correspond to the fiber axis. This point is illustrated in Figure 9 where some (hk0) planes are represented.
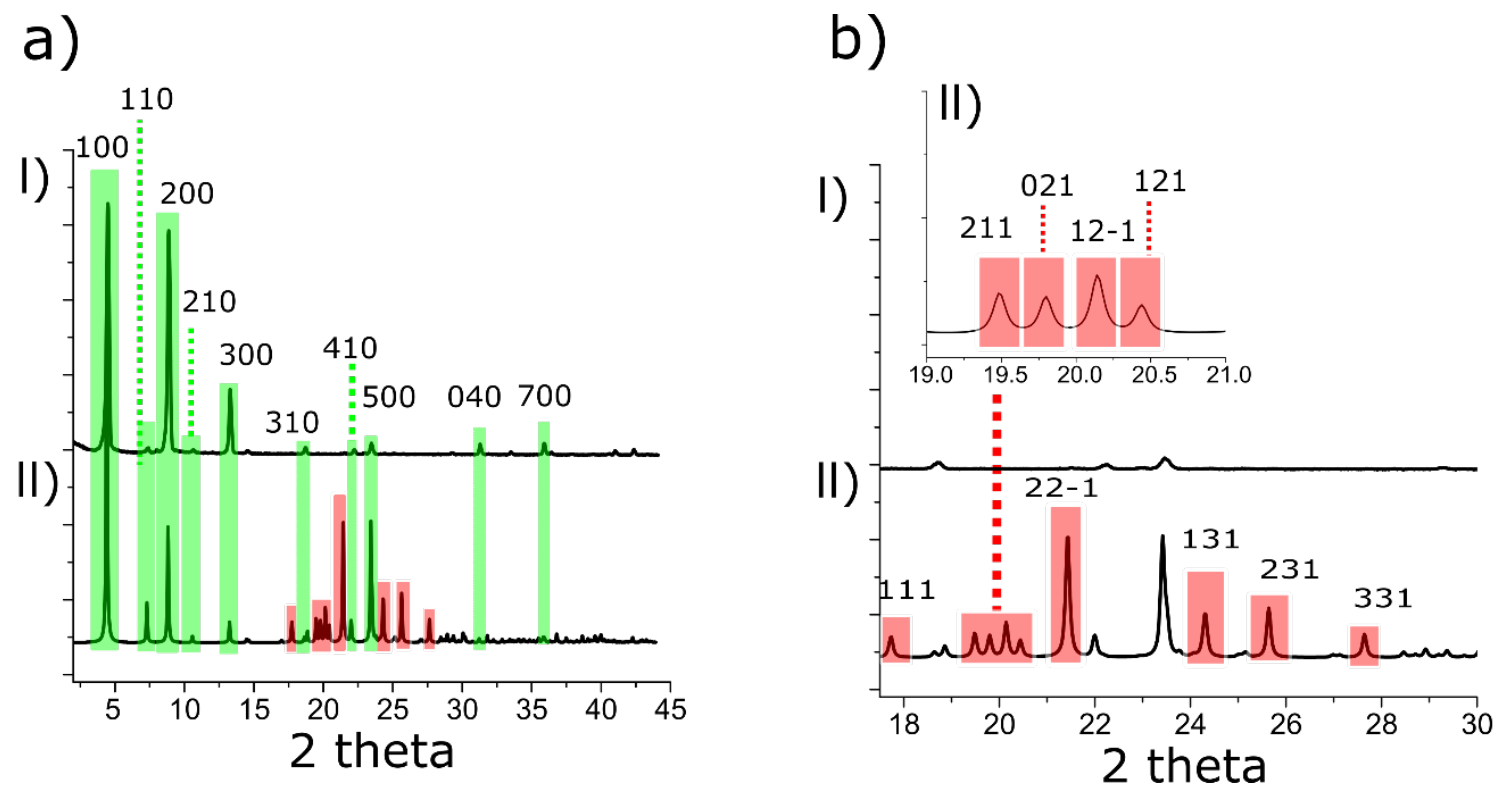

Figure 8. Comparison between the X-ray patterns of oriented (top) and randomized (bottom) gel fibers of Th12.a) full range pattern. b) Close-up in the 17.5 - 30 2-theta range; the inset is a further zoom on the 19.0 - 21.0 2-theta range. Green highlighting corresponds to the diffraction peaks present in both X-ray patterns. Red highlighting corresponds to diffraction peaks only present in randomized fibers. 


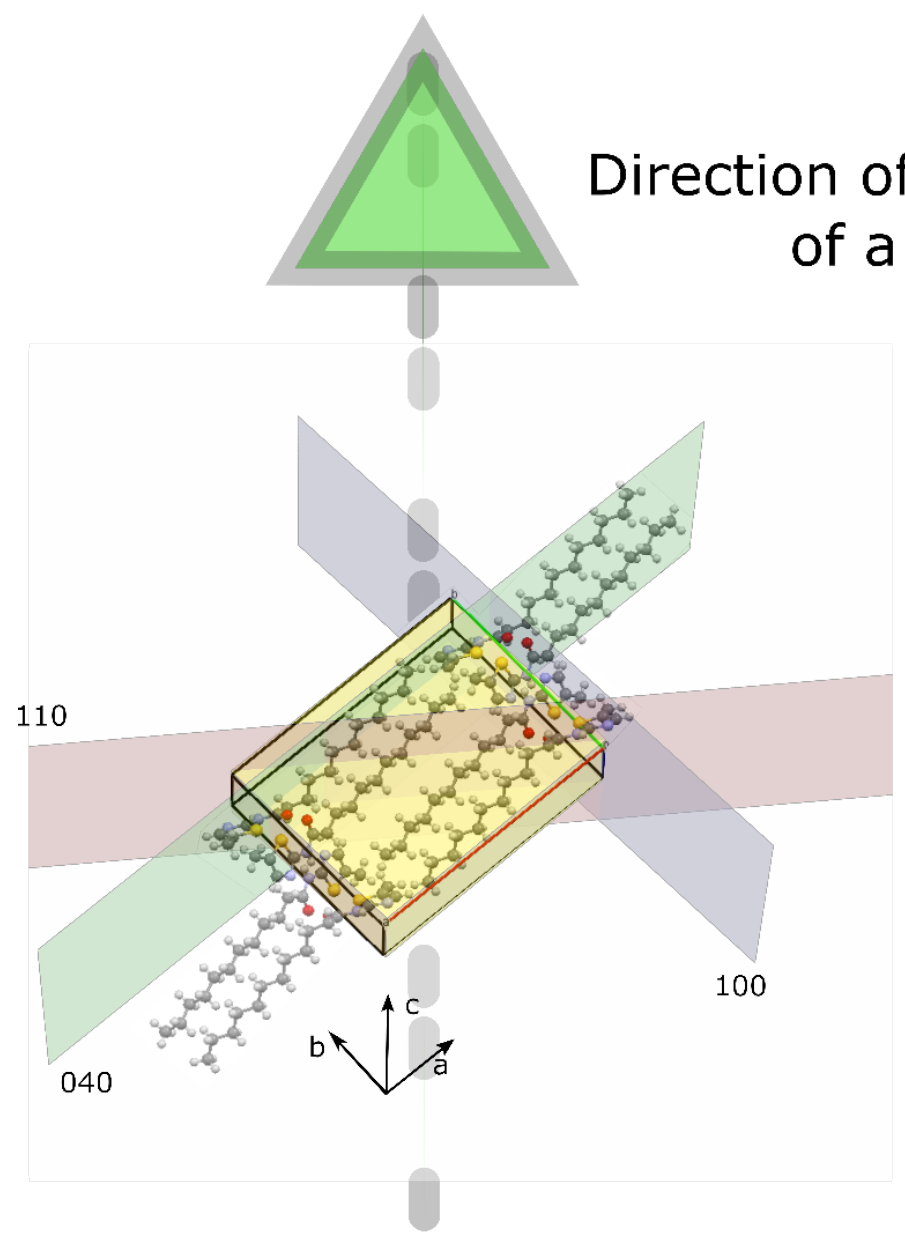

Figure 9. Representation of the diffraction planes observed in the X-ray patterns of the oriented fibers.

A further proof of the growth direction was given by calculations with the Morphology module of Materials Studio. Two simulation methods were used: the Bravais-Friedel-Donnay-Harker ${ }^{36,37}$ (BFDH) and the Growth Morphology Method ${ }^{38,39}$ (GMM). The BFDH method is based on purely geometrical rules using crystal lattice and symmetry elements, with the growth rate of a given face inversely proportional to the thickness of the molecular layer added to it. Even though energy 
effects are neglected, BFDH is often reliable for weakly interacting molecules, and is helpful in showing prevailing faces in crystal growth. In the GMM method, the growth rate of a face is proportional to the attachment energy (Eatt), i.e. the potential energy per unit cell gained when a new molecular layer is attached to the face. Faces with the strongest attachment energies grow fastest and have the lowest morphological dominance.

With GMM, we find that the crystal habit is elongated along the [001] direction, with $\{100\}$, $\{110\}$ (and $\{020\}$ for Th12) lateral faces, and is capped by $\{11-1\}$ and $\{011\}$ faces (Table 2 ). These results confirm the X-ray measurements on oriented fibers, where the signal of planes parallel to the long axis, here the $\{\mathrm{hk} 0\}$ planes, is intensified, in contrast to the signal of planes tilted with respect to the long axis, i.e. planes with $\mathrm{L}$ different from 0 , which are attenuated. A similar crystal habit was found with BFDH (see SI), suggesting that simple geometry constrains are sufficient to induce a high anisotropy of the crystal, in a system where it is not straightforward to pinpoint strong intermolecular interactions along a particular direction (the hydrogen bonds lead to the formation of dimers, not rows of H-bonded molecules).

As the length of the alkyl chain only affects the $a$ cell parameter, only faces with a dominant $\mathrm{h}$ character grow slower as the molecules get longer. As a result, from Th12 to Th18, the facet area of $\{100\}$ increases with respect to that of $\{110\}$, from 1.3:1 to 1.9:1 with BFDH, and from 0.6:1 to $0.7: 1$ with GMM, and the $\{020\}$ face disappears from Th14 on. In addition, the anisotropy grows slightly from Th12 to Th18, with an aspect ratio evolving from 4.3 to 5.7 with BFDH, and from 2.5 to 2.9 with GMM. This stronger anisotropy could be the reason why the radius of the gelation sphere increases with the length of the alkyl chain (Figure 3a). Indeed, more anisotropic fibers have a higher tendency to form a percolating network. 
Due to symmetry operations, there are two or four ways to build a face, depending on the part of the molecule that is exposed to the environment; this influences the surface stability and thus the facet growth. With the GMM method, the face covering, i.e., the molecular groups present at the outer surface, that grows slowest is always selected as the limiting factor. In Table 2, the characteristics of the relevant faces relate to the most stable covering, except for the $\{100\}$ faces, where information for the two possible coverings (alkyl chains or thiazole groups) are indicated. The $\{100\}$ faces can be covered either by alkyl chains or thiazole groups protruding perpendicular to the surface. The alkyl chain covering and its Eatt are strongly affected by the molecular size, as the length of alkyl chain dangling in the environment increases (from Th12 to Th18, Eatt evolves from -71.3 to $-104.2 \mathrm{kcal} / \mathrm{mol}$ ), while the thiazole covering and its Eatt are not (from Th12 to Th18, Eatt drifts only from -82.3 to $-83.3 \mathrm{kcal} / \mathrm{mol}$, respectively). As a result, the face covering could change depending on the size of the alkyl chain. Here, the face covering appears more stable with alkyl chains below Th16, while from Th16 on it is the thiazole termination that is most stable. However, we must keep in mind that in this approach the effects of the relaxation of molecules and interactions with the solvent were neglected. The relaxation of the long dangling alkyl chains could significantly reduce the attachment energies, slow down the growth of the $\{100\}$ faces, and further increase the anisotropy of the fibers. The $\{100\}$ faces could also adapt their covering in response to the environment, either exposing polar groups or apolar groups. Furthermore, apolar groups present at the surface could either extend into the solvent or adsorb on the crystal face.

These protruding molecular groups are typical of $\{100\}$ faces and could help stabilizing the fibers; for other crystal faces, they are less conspicuous or do not exist at all, e.g. for $\{020\}$, where both thiazole groups and alkyl chains lie flat at the surface. Due to this orientation, the relative occupancy of the $\{020\}$ surfaces by the apolar alkyl chains increases with the size of the molecule. 
This increase of the apolar character is also observed for the four possible coverings of the dominant $\{110\}$ faces, as the molecules are tilted with respect to the surface (see SI).

In summary, our calculations show that the crystal habit of Th12 to Th18 are very similar and that when the alkyl chain length is increased, the proportion of polar and apolar groups at the surface changes, likely resulting in a gradual increase in the contact between apolar parts of the fiber and the solvent. We propose that this is the reason why the gelation sphere shifts regularly toward the less polar region of the Hansen space from Th12 to Th18.

Table 2. List of relevant faces of thiazole based LMWG obtained by the GMM method, sorted out by attachment energy (Eatt in $\mathrm{kcal} / \mathrm{mol}$ ).

\begin{tabular}{|c|c|c|c|c|}
\hline & & Multiplicity & Eatt total & $\%$ total facet area \\
\hline \multirow{6}{*}{ Th12 } & $\{100\}$-alk & 2 & -71.3 & 27.8 \\
\hline & $\{100\}$-th & 2 & -82.3 & - \\
\hline & $\{110\}$ & 4 & -76.6 & 44.0 \\
\hline & $\{020\}$ & 2 & -87.2 & 4.8 \\
\hline & $\{11-1\}$ & 4 & -144.8 & 16.1 \\
\hline & $\{011\}$ & 4 & -154.1 & 7.3 \\
\hline \multirow{6}{*}{ Th14 } & $\{100\}$-alk & 2 & -82.3 & 26.2 \\
\hline & $\{100\}$-th & 2 & -82.6 & - \\
\hline & $\{110\}$ & 4 & -82.3 & 50.9 \\
\hline & $\{020\}$ & 2 & -98.1 & - \\
\hline & $\{11-1\}$ & 4 & -161.1 & 14.6 \\
\hline & $\{011\}$ & 4 & -170.4 & 8.3 \\
\hline \multirow{3}{*}{ Th16 } & $\{100\}$-th & 2 & -82.9 & 29.9 \\
\hline & $\{100\}$-alk & 2 & -93.3 & - \\
\hline & $\{110\}$ & 4 & -88.0 & 48.3 \\
\hline
\end{tabular}




\begin{tabular}{lllcc}
\hline \multirow{2}{*}{020} & 2 & -108.9 & - \\
\cline { 2 - 5 } & $\{11-1\}$ & 4 & -177.3 & 13.2 \\
\cline { 2 - 5 } Th18 & $\{100\}$-th & 2 & -186.6 & 8.6 \\
\hline \multirow{2}{*}{100} -alk & 2 & -83.3 & 33.2 \\
\cline { 2 - 5 } & $\{110\}$ & 4 & -104.2 & - \\
\cline { 2 - 5 } & $\{020\}$ & 2 & -119.8 & 45.9 \\
\cline { 2 - 5 } & $\{11-1\}$ & 4 & -193.5 & 12.1 \\
\hline$\{011\}$ & 4 & -202.9 & 8.8
\end{tabular}

The $\{100\}$-th and $\{100\}$-alk notations correspond to thiazole-terminated and alkyl chainterminated $\{100\}$ faces, respectively.

[001]

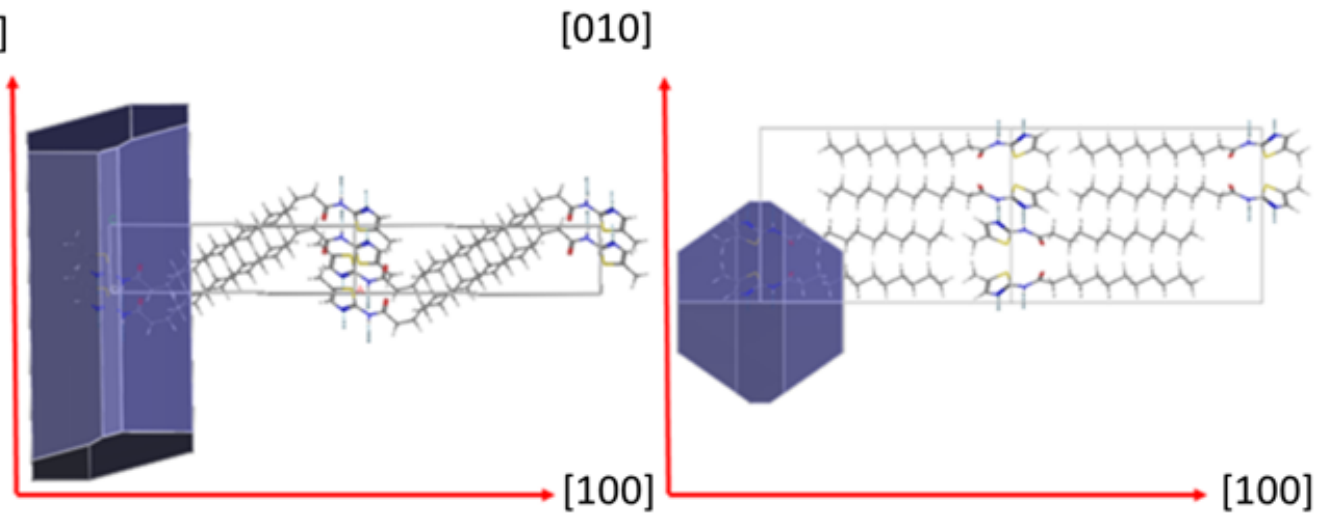

Figure 10. Crystal habit of Th12 built from the Growth Morphology Method. Views along the 010 and 001 directions, and orientation of the molecules with respect to the macroscopic crystal.

\section{Conclusion}

This study presents an in-depth crystallographic analysis of the molecular packing and crystal habit of a set of thiazole-based gelators. In terms of solid-state structure, we show that the four molecules differing only in the length of an alkyl chain crystallize in the same symmetry group, 
and with the same crystal habit. In terms of gelation behavior, the increase in alkyl chain length in these gelators: (i) increases the size of the gelation sphere in the Hansen space and (ii) gradually shifts its center. We attribute the first effect to an increased anisotropy for fiber growth when the alkyl chain length is increased. We attribute the second effect to the fact that, since all the studied gelators share the same molecular packing, an increasing length of the alkyl chain gradually increases the contact between apolar parts of the fiber and the solvent and therefore reduces the relative influence of polar and hydrogen bond interactions.

\section{Acknowledgements}

This work was supported by the French Agence Nationale de la Recherche (project ANR-16CE08-0012 HSPgel). Research in Mons was supported by the UMONS research council and FNRS, through the EOS 2Dto3D project and the 'Consortium des Equipements de Calcul Intensif' (CECI - grant No. 2.5020.11). We acknowledge the provision of facilities and technical support by Aalto University at OtaNano - Nanomicroscopy Center (Aalo-NMC).

\section{Notes and References}

(1) Terech, P.; Weiss, R. G. Low Molecular Mass Gelators of Organic Liquids and the Properties of Their Gels. Chem. Rev. 1997, 97 (8), 3133-3160.

(2) George, M.; Weiss, R. G. Molecular Organogels. Soft Matter Comprised of LowMolecular-Mass Organic Gelators and Organic Liquids. Acc. Chem. Res. 2006, 39 (8), 489_ 497.

(3) Dastidar, P. Supramolecular Gelling Agents: Can They Be Designed? Chem. Soc. Rev. 2008, 37 (12), 2699.

(4) Raynal, M.; Bouteiller, L. Organogel Formation Rationalized by Hansen Solubility Parameters. Chem. Commun. 2011, 47 (29), 8271. 
(5) Bonnet, J.; Suissa, G.; Raynal, M.; Bouteiller, L. Organogel Formation Rationalized by Hansen Solubility Parameters: Dos and Don'ts. Soft Matter 2014, 10 (18), 3154.

(6) Rosa Nunes, D.; Raynal, M.; Isare, B.; Albouy, P.-A.; Bouteiller, L. Organogel Formation Rationalized by Hansen Solubility Parameters: Improved Methodology. Soft Matter 2018, 14 (23), 4805-4809.

(7) Gao, J.; Wu, S.; Rogers, M. A. Harnessing Hansen Solubility Parameters to Predict Organogel Formation. J. Mater. Chem. 2012, 22 (25), 12651.

(8) Xu, H.; Song, J.; Tian, T.; Feng, R. Estimation of Organogel Formation and Influence of Solvent Viscosity and Molecular Size on Gel Properties and Aggregate Structures. Soft Matter 2012, 8 (12), 3478.

(9) Huang, Y.; Yuan, Y.; Tu, W.; Zhang, Y.; Zhang, M.; Qu, H. Preparation of Efficient Organogelators Based on Pyrazine-2,5-Dicarboxylic Acid Showing Room Temperature Mesophase. Tetrahedron 2015, 71 (21), 3221-3230.

(10) Lai, T. L.; Canevet, D.; Avarvari, N.; Sallé, M. Internal Probing of the Supramolecular Organization of Pyrene-Based Organogelators. Chem. - An Asian J. 2016, 11 (1), 81-85.

(11) Wang, T.; Yu, X.; Li, Y.; Ren, J.; Zhen, X. Robust, Self-Healing, and MultistimuliResponsive Supergelator for the Visual Recognition and Separation of Short-Chain Cycloalkanes and Alkanes. ACS Appl. Mater. Interfaces 2017, 9 (15), 13666-13675.

(12) Xiao, T.; Zhang, X.; Wu, J.; Yang, J.; Yang, Y. Aliphatic-Alcohol-Induced Opaque-toTransparent Transformation and Application of Solubility Theory in a Bis-Dipeptide-Based Supramolecular Gel. Chempluschem 2017, 82 (6), 879-887.

(13) Lan, Y.; Corradini, M. G.; Liu, X.; May, T. E.; Borondics, F.; Weiss, R. G.; Rogers, M. A. Comparing and Correlating Solubility Parameters Governing the Self-Assembly of Molecular Gels Using 1,3:2,4-Dibenzylidene Sorbitol as the Gelator. Langmuir 2014, 30 (47), 14128-14142.

(14) Yin, Y.; Gao, Z.; Bao, Y.; Hou, B.; Hao, H.; Liu, D.; Wang, Y. Gelation Phenomenon during 
Antisolvent Crystallization of Cefotaxime Sodium. Ind. Eng. Chem. Res. 2014, 53 (3), 1286-1292.

(15) Zhang, M.; Selvakumar, S.; Zhang, X.; Sibi, M. P.; Weiss, R. G. Structural and Solubility Parameter Correlations of Gelation Abilities for Dihydroxylated Derivatives of LongChain, Naturally Occurring Fatty Acids. Chem. - A Eur. J. 2015, 21 (23), 8530-8543.

(16) Liu, C.; Corradini, M.; Rogers, M. A. Self-Assembly of 12-Hydroxystearic Acid Molecular Gels in Mixed Solvent Systems Rationalized Using Hansen Solubility Parameters. Colloid Polym. Sci. 2015, 293 (3), 975-983.

(17) Diehn, K. K.; Oh, H.; Hashemipour, R.; Weiss, R. G.; Raghavan, S. R. Insights into Organogelation and Its Kinetics from Hansen Solubility Parameters. Toward a Priori Predictions of Molecular Gelation. Soft Matter 2014, 10 (15), 2632.

(18) Lan, Y.; Corradini, M. G.; Rogers, M. A. Do Molecular Gelators Cluster in Hansen Space? Cryst. Growth Des. 2014, 14 (9), 4811-4818.

(19) Aparicio, F.; García, F.; Sánchez, L. Supramolecular Polymerization of C3-Symmetric Organogelators: Cooperativity, Solvent, and Gelation Relationship. Chem - A Eur. J. 2013, $19(9), 3239-3248$.

(20) Edwards, W.; Smith, D. K. Dynamic Evolving Two-Component Supramolecular Gels Hierarchical Control over Component Selection in Complex Mixtures. J. Am. Chem. Soc. $\mathbf{2 0 1 3}, 135$ (15), 5911-5920.

(21) Wu, S.; Gao, J.; Emge, T. J.; Rogers, M. A. Influence of Solvent on the Supramolecular Architectures in Molecular Gels. Soft Matter 2013, 9 (25), 5942-5950.

(22) Edelsztein, V. C.; Mac Cormack, A. S.; Ciarlantini, M.; Di Chenna, P. H. Self-Assembly of 2,3-Dihydroxycholestane Steroids into Supramolecular Organogels as a Soft Template for the in-Situ Generation of Silicate Nanomaterials. Beilstein J. Org. Chem. 2013, 9, 18261836.

(23) Ando, T.; Ito, K. Gelation Properties of Poly(Aryl Ether) Dendrons Bearing Long Alkyl 
Chains. J. Incl. Phenom. Macrocycl. Chem. 2014, 80 (3-4), 285-294.

(24) He, S.; Zhao, H.; Guo, X.; Xu, X.; Zhou, X.; Liu, J.; Xing, Z.; Ye, L.; Jiang, L.; Chen, Q.; et al. The Readout of Base-Pair Information in Adenine-Thymine $\alpha-D$ Arabinonucleosides. Chem. - A Eur. J. 2014, 20 (47), 15473-15481.

(25) Tong, C.; Fan, K.; Niu, L.; Li, J.; Guan, X.; Tao, N.; Shen, H.; Song, J. Application of Solubility Parameters in a D-Sorbitol-Based Organogel in Binary Organic Mixtures. Soft Matter 2014, 10 (5), 767-772.

(26) Shen, H.; Niu, L.; Fan, K.; Li, J.; Guan, X.; Song, J. Application of Solubility Parameters in 1,3:2,4-Bis(3,4- Dimethylbenzylidene)Sorbitol Organogel in Binary Organic Mixtures. Langmuir 2014, 30 (30), 9176-9182.

(27) Yan, N.; Xu, Z.; Diehn, K. K.; Raghavan, S. R.; Fang, Y.; Weiss, R. G. Pyrenyl-LinkerGlucono Gelators. Correlations of Gel Properties with Gelator Structures and Characterization of Solvent Effects. Langmuir 2013, 29 (2), 793-805.

(28) Edelsztein, V. C.; Mac Cormack, A. S.; Ciarlantini, M.; Di Chenna, P. H. Self-Assembly of 2,3-Dihydroxycholestane Steroids into Supramolecular Organogels as a Soft Template for the in-Situ Generation of Silicate Nanomaterials. Beilstein J. Org. Chem. 2013, 9, 18261836 .

(29) Yan, N.; Xu, Z.; Diehn, K. K.; Raghavan, S. R.; Fang, Y.; Weiss, R. G. How Do Liquid Mixtures Solubilize Insoluble Gelators? Self-Assembly Properties of Pyrenyl-LinkerGlucono Gelators in Tetrahydrofuran-Water Mixtures. J. Am. Chem. Soc. 2013, 135 (24), 8989-8999.

(30) Zhang, M.; Selvakumar, S.; Zhang, X.; Sibi, M. P.; Weiss, R. G. Structural and Solubility Parameter Correlations of Gelation Abilities for Dihydroxylated Derivatives of LongChain, Naturally Occurring Fatty Acids. Chem. - A Eur. J. 2015, 21 (23), 8530-8543.

(31) Li, Z.; Huang, Y.; Fan, D.; Li, H.; Liu, S.; Wang, L. Synthesis and Properties of Novel Organogelators Functionalized with 5-Iodo-1,2,3-Triazole and Azobenzene Groups. Front. 
Chem. Sci.Eng. 2016, 10 (4), 552-561.

(32) Singh, A.; Auzanneau, F.-I. I.; Corradini, M. G.; Grover, G.; Weiss, R. G.; Rogers, M. A. Molecular Nuances Governing the Self-Assembly of 1,3:2,4-Dibenzylidene-D-Sorbitol. Langmuir 2017, 33 (41), 10907-10916.

(33) Bonnet, J.; Suissa, G.; Raynal, M.; Bouteiller, L. Organogel Formation Rationalized by Hansen Solubility Parameters: Influence of Gelator Structure. Soft Matter 2015, 11 (11), 2308-2312.

(34) Yadav, P.; Kour, D.; Gupta, V. K.; Rajnikant; Ballabh, A. Probing the Role of Weaker Interactions in Immobilization of Solvents in a New Class of Supramolecular Gelator. RSC $A d v .2013,3$ (22), 8417-8421.

(35) Yadav, P.; Ballabh, A. Odd-Even Effect in a Thiazole Based Organogelator: Understanding the Interplay of Non-Covalent Interactions on Property and Applications. New J. Chem. 2015, $39(1), 721-730$.

(36) Bravais, A. Etudes Crystallographiques, Academie des Sciences: Paris (1913).

(37) Friedel, G. Etudes sur la loi de Bravais. Bull. Soc. Fr. Mineral., 30, 326 (1907).

(38) Berkovitch-Yellin, Z. Toward an ab initio derivation of crystal morphology. J. Am. Chem. Soc., 107, 8239 (1985).

(39) Docherty, R.; Clydesdale, G.; Roberts, K. J.; Bennema, P. Application of Bravais-FriedelDonnay-Harker attachment energy and Ising models to predicting and understanding the morphology of molecular crystals. J. Phys. D: Appl. Phys., 24, 89 (1991).

(40) Hansen, C. M. Hansen Solubility Parameters A User's Handbook, Second Edition; 2007.

(41) Abbott, S.; Hansen, C. M. Hansen Solubility Parameters in Practice; 2008.

(42) Atiq, S.; Ullah, Z.; Naseem, S. Indexing the Diffraction Patterns and Investigating the Crystal Structure of Pb-Doped Strontium Ferrites. J. Sci.Res. 2013, 5 (2), 235-244. 
(43) Rodríguez-Carvajal, J. Recent Advances in Magnetic Structure Determination by Neutron Powder Diffraction. Phys. B Condens. Matter 1993, 192 (1-2), 55-69.

(44) BIOVIA, D. S. Materials Studio. 2011.

(45) No gelation sphere could be determined for Th8 because only two liquids form a gel. 